\title{
Recombinant Human Adenovirus Type 5 $\mathrm{H} 101$
}

National Cancer Institute

\section{Source}

National Cancer Institute. Recombinant Human Adenovirus Type 5 H101. NCI

Thesaurus. Code C107504.

A replication selective, recombinant, E1B and partial E3 gene deleted form of human adenovirus type 5, with potential antineoplastic activity. Upon intratumoral injection of recombinant human adenovirus type 5, the adenovirus selectively replicates in cancer cells while preventing viral replication in normal, healthy cells. This induces a selective adenovirus-mediated cytotoxicity in cancer cells, which leads to cancer cell lysis. In addition, viral spread to adjacent cells, following lysis of infected cells, may activate the immune system to kill the infected tumor cells. The E1B protein causes p53 inactivation, which promotes viral replication; deletion of E1B allows for p53 activation in normal cells, which prevents viral replication in normal, healthy cells. The mutation and subsequent inactivation of p53 in cancer cells enables the E1B-deleted adenovirus to selectively replicate in cancer cells. Partial deletion of E3, encoding the adenovirus death protein, enhances the safety profile of the administered adenovirus. 